\title{
Physcion Protects Rats Against Cerebral Ischemia-Reperfusion Injury via Inhibition of TLR4/NF-kB Signaling Pathway
}

This article was published in the following Dove Press journal:

Drug Design, Development and Therapy

\section{Xiaobo Dong* \\ Lei Wang* \\ Guangrong Song \\ Xu Cai \\ Wenxin Wang \\ jiaqi Chen \\ Gesheng Wang}

The Third Department of Encephalopathy, Dongfang Hospital Beijing University of Chinese Medicine, Beijing I00078, People's Republic of China

*These authors contributed equally to this work
Correspondence: Gesheng Wang

The Third Department of Encephalopathy, Dongfang Hospital Beijing University of Chinese Medicine, No. 6

Zone I Fangxingyuan, Fangzhuang, Fengtai

District, Beijing 100078, People's Republic of China

Email wanggesh@sina.com
Background: Ischemic stroke (IS) is characterized by the rapid loss of brain function due to ischemia. Physcion has been found to have a neuroprotective effect against cerebral ischemia-reperfusion (I/R) injury. However, the mechanism by which physcion regulates cerebral $\mathrm{I} / \mathrm{R}$ injury remains largely unknown.

Methods: An oxygen-glucose deprivation/reperfusion (OGD/R) model in SH-SY5Y cells and a rat cerebral ischemia-reperfusion (I/R) model were established, respectively. CCK-8 and flow cytometry assays were used to detect the viability and apoptosis of SH-SY5Y cells. Moreover, enzyme-linked immunosorbent assay (ELISA) was used to measure the levels of SOD, MDA, GSH-Px, TNF- $\alpha$, IL-1 $\beta$, IL-6 and IL-10 in the supernatant of SH-SY5Y cells. Meanwhile, Western blot assay was used to detect the expressions of TLR4, p-p65 and p-IкB in SH-SY5Y cells and I/R rats.

Results: In this study, physcion treatment significantly rescued OGD/R-induced neuronal injury. In addition, physcion decreased inflammatory response in SH-SY5Y cells after OGD/R insult, as shown by the decreased levels of the pro-inflammatory factors TNF- $\alpha$, IL-1 $\beta$, IL- 6 and IL-10. Moreover, physcion attenuated the oxidative stress in OGD/R-treated SY-SY5Y cells, as evidenced by the increased SOD and GSH levels and the decreased ROS and MDA levels. Meanwhile, physcion significantly reduced cerebral infarction, attenuated neuronal injury and apoptosis in $\mathrm{I} / \mathrm{R}$

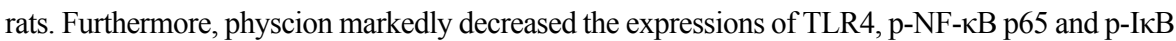
in the brain tissues of rats subjected to I/R and in SH-SY5Y cells exposed to OGD/R.

Conclusion: In conclusion, our study indicated that physcion protected neuron cells against $\mathrm{I} / \mathrm{R}$ injury in vitro and in vivo by inhibition of the TLR4/NF-kB pathway; thus, physcion might serve as a promising therapeutic candidate for IS.

Keywords: stroke, ischemia-reperfusion injury, physcion, NF- $\mathrm{B}$

\section{Introduction}

Stroke is the third leading cause of mortality worldwide, which is an important cause of long-term disability. ${ }^{1}$ Ischemic stroke (IS) is a common neurovascular disease in the central nervous system (CNS), which commonly accounts for about $80 \%$ of all stroke cases. $^{2}$ Recanalization therapies are known as the primary treatments for IS, which can replenish oxygen, nutrients, and remove toxic metabolites. ${ }^{3}$ However, restoration of blood flow unavoidably generates pathological damage in the ischemic brain tissue, ultimately leads to the cerebral ischemia/reperfusion (I/R) injury. ${ }^{4}$ Since numerous mechanisms, such as cell toxicity, apoptosis, oxidative stress and inflammation are found to be involved in cerebral I/R injury, ${ }^{5}$ the pathogenesis of stroke is extremely 
complex. ${ }^{6}$ Therefore, the development of new drugs may be beneficial for the treatment of IS.

Nuclear factor-kappa B (NF- $\mathrm{B})$, a transcription factor, has been found to participate in several cell functions including hematogenesis, inflammation, immune response, cell apoptosis. ${ }^{7}$ In addition, NF- $\kappa \mathrm{B}$ has been shown to regulate the production of multiple pro-inflammatory factors, including TNF- $\alpha$, IL-6 and IL- $1 \beta$ in neuronal cells. ${ }^{8}$ Liu et al indicated that pterostilbene could attenuate neuronal oxidative damage following $\mathrm{I} / \mathrm{R}$ injury by inactivation of NF- $\kappa$ B. ${ }^{9}$

Physcion has an anthraquinone chemical structure deriving from the rhizome of the Chinese herbs medicine Radix et Rhizoma Rhei. ${ }^{10}$ In addition, physcion has been found to exhibit strong anti-inflammatory, anti-bacterial, and anti-tumor properties. ${ }^{11,12} \mathrm{Li}$ et al indicated that physcion exerted a potential anti-ischemic effect in cerebral ischemia reperfusion (I/R) model in rats. ${ }^{13}$ Meanwhile, feng et al indicated that rhubarb anthraquinones were easier to be absorbed in $\mathrm{I} / \mathrm{R}$ rats, which proves the rationality of using it in stroke. ${ }^{14}$ However, the function and regulatory mechanism of physcion in IS remains poorly unclear. Therefore, this study aimed to investigate the role of physcion during the neuronal damage after cerebral I/R.

\section{Materials and Methods}

\section{Cell Culture}

SH-SY5Y cell line was obtained from the Type Culture Collection of the Chinese Academy of Sciences (Shanghai, China). The cells were cultured in Dulbecco's Modified Eagle Medium (DMEM, Thermo Fisher Scientific, Inc., Waltham, MA, USA) supplemented with $10 \%$ heatinactivated fetal bovine serum (Thermo Fisher Scientific) and penicillin/streptomycin, and incubated with $5 \% \mathrm{CO}_{2}$ at $37^{\circ} \mathrm{C}$.

\section{Oxygen-Glucose Deprivation/ Reoxygenation (OGD/R) Model}

For OGD treatment, the normal culture medium was replaced with glucose-free DMEM medium, and cells were exposed to hypoxic conditions $\left(5 \% \mathrm{CO}_{2}, 95 \% \mathrm{~N}_{2}\right)$ at $37^{\circ} \mathrm{C}$ for $4 \mathrm{~h} .{ }^{15}$ After that, the cells were reoxygenated under normoxic conditions $\left(5 \% \mathrm{CO}_{2}\right.$ and $95 \%$ air) and cultured in a glucose-containing culture medium plus $10 \%$ FBS for $24 \mathrm{~h}$. There are there groups: control group, an $\mathrm{OGD} / \mathrm{R}$ group and an $\mathrm{OGD} / \mathrm{R}+$ physcion group (physcion intervention group; 10, 20, or $40 \mu \mathrm{M}$ physcion were added). In the control group, the cells were incubated in DMEM supplemented with serum and glucose in a normoxic atmosphere. In the OGD/R group, cells were subjected to OGD/R. In the OGD/R + physcion group, cells were treated with OGD for $4 \mathrm{~h}$ and then returned to normal culture conditions with physcion (10, 20 , or $40 \mu \mathrm{M}$ ) for another $24 \mathrm{~h}$.

\section{Cell Survival Assay}

Cell viability was determined using the Cell Counting Kit8 (CCK-8) assay (Dojindo Laboratories, Kumamoto, Japan). Cells were seeded in a 96-well plate at a density of $5 \times 10^{3}$ cells/well, and then $10 \mu \mathrm{L}$ of CCK-8 reagent was added into each well and incubated for another $2 \mathrm{~h}$ at $37^{\circ} \mathrm{C}$ according to the manufacturer's instructions. The optical density (OD) value of each well at $450 \mathrm{~nm}$ was assessed by an AMR-100 automatic enzyme analyzer (Allsheng, Hangzhou, China).

\section{Cell Apoptosis Assay}

Annexin V-FITC/PI Apoptosis Detection Kit (Thermo Fisher Scientific) was used for the detection of apoptotic cells according to the manufacturer's protocol. Cells were washed twice with pre-cooled PBS, and then stained with propidium iodide (PI; $10 \mu \mathrm{L})$ and Annexin V-FITC (10 $\mu \mathrm{L}$ ) for $15 \mathrm{~min}$ in the darkness. Subsequently, a FACSort flow cytometer (BD Biosciences, Mountain View, CA, USA) was applied to measure the percentage of the apoptotic cells.

\section{Intracellular Reactive Oxygen Species (ROS) Detection}

SH-SY5Y cells were stained with $10 \mu \mathrm{M}$ of 2,7-dichlorofluorescein diacetate (DCFH-DA, Beyotime, Haimen, China) in the dark for $20 \mathrm{~min}$. After that, cells were collected and suspended in $500 \mu \mathrm{L}$ of PBS. Subsequently, the fluorescence intensity was determined using a FACSort flow cytometer (BD Biosciences).

\section{ELISA Assay}

The cell culture supernatant samples were collected from SH-SY5Y cells. Then, superoxide dismutase (SOD), malondialdehyde (MDA), glutathione peroxidase (GSH-Px), interleukin-1 $\beta$ (IL-1 $\beta$ ), tumor necrosis factor- $\alpha$ (TNF- $\alpha$ ), interleukin-10 (IL-10), interleukin-6 (IL-6), and macrophage chemoattractant protein 1 (MCP-1) assay kits (\#A001-3, A003-1, A005-1, H002, H052, H009, H007, 
H115; Jiancheng, Bioengineering Institute, Nanjing, China) were used to measure SOD, MDA, GSH-Px, IL$1 \beta$, TNF- $\alpha$, IL-10, IL-6, and MCP-1 contents according to the manufacturer's protocol.

\section{Western Blot Assay}

Total protein concentration was detected using the BCA Protein Assay kit (Beyotime). Protein samples of $40 \mu \mathrm{g}$ per lane were separated by $10 \%$ sodium dodecyl sulfatepolyacrylamide gel electrophoresis (SDS-PAGE), and then transferred onto a PVDF membrane (Millipore, Billerica, MA, USA). After that, the membrane was blocked with $5 \%$ non-fat milk for $1 \mathrm{~h}$ at room temperature, and then incubated overnight at $4{ }^{\circ} \mathrm{C}$ with the following primary antibodies against: toll-like receptor 4 (TLR4; 1:1000, Abcam), phospho-NF-кB p65 (p-p65; 1:1000, Abcam),

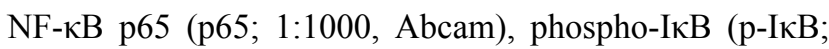
1:1000, Abcam), IкB (1:1000, Abcam), $\beta$-actin (1:1000, Abcam), and further incubated with HRP-labeled Goat Anti-Rabbit IgG $(\mathrm{H}+\mathrm{L})$ antibody (Abcam) at room temperature for $1 \mathrm{~h}$. Finally, the protein bands were visualized by an enhanced chemiluminescent (ECL) reagent (Thermo Fisher Scientific).

\section{Animal Study}

SD rats ( 8 weeks old) were obtained from Shanghai SLAC Animal Center. Middle cerebral artery occlusion (MCAO) was performed in accordance with methods described by Lee et al. ${ }^{16}$ Briefly, rats were anaesthetized with ketamine $(30 \mathrm{mg} / \mathrm{kg})$ and xylazine cocktail $(20 \mathrm{mg} / \mathrm{kg})$. After that, a 7-0 surgical nylon monofilament with a rounded tip was inserted into the internal carotid artery (ICA), then occluding the middle cerebral artery (MCA). After $2 \mathrm{~h}$, nylon monofilament was retracted, and reperfusion began. Then, the rats were allowed to recover for 7 days. These rats were randomly divided into the four groups $(n=18$ per group): Sham group; I/R group; I/R + physcion_20 mg/kg group; I/R + physcion_40 mg/kg group. Rats were treated with physcion (20 or $40 \mathrm{mg} / \mathrm{kg}$ ) via oral gavage after $I / R$ every day. The animal used and experimental protocols were approved by the Animal care Committee of the Dongfang Hospital Beijing University of Chinese Medicine (No. BUCM-20,190,822). All animals were treated in accordance with the National Institutes of Health Guide for Care and Use of Laboratory Animals. Physcion (purity: $>98.0 \%$ ) was obtained from MCE (cat. no. HYN0108; Med Chem Express, Monmouth Junction, NJ, USA).

\section{Triphenyltetrazolium Chloride (TTC) Staining}

TTC staining was performed as described previously. ${ }^{16}$ The brain tissues ( $\mathrm{n}=6$ per group) were cut into six slices with $1 \mathrm{~mm}$ thickness, and the sections were stained with $2 \%$ TTC (Sigma-Aldrich, St. Louis, MO, USA) for $20 \mathrm{~min}$ at $37^{\circ} \mathrm{C}$. The ipsilateral infarct area was analyzed by ImageJ software. Infarcted area was expressed as pale unstained.

\section{Determination of Cerebral Water Content}

The brain tissues ( $\mathrm{n}=6$ per group) were dried at $105^{\circ} \mathrm{C}$ for $24 \mathrm{~h}$ to measure the dry weight. Then, brain water content (\%) was calculated as ((wet weight - dry weight)/wet weight $\times 100 \%$ ).

\section{Neurological Severity Scorers (NSS)}

The neurological function was assessed by NSS described by Chen et al. ${ }^{17}$ NSS was conducted by an investigator who was blinded to animal treatments.

\section{Histological Examination}

The same brain area of rat in each group $(n=3$ per group) was used to perform the hematoxylin and eosin (H\&E) staining and TUNEL assays. The brain tissues were fixed in $4 \%$ paraformaldehyde at $4^{\circ} \mathrm{C}$, and then embedded in paraffin, and cut into $4 \mu \mathrm{m}$-thick sections. The sections were stained with hematoxylin and eosin, and visualized via a Leica DM 5000B microscopy (Leica Microsystems, Wetzlar, Germany). The TUNEL assay was conducted by using a In Situ Cell Death Detection Kit (Roche; Indianapolis, IN, USA). The image of TUNEL-positive cells in brain tissues were captured by a Leica DM 5000B microscopy (Leica Microsystems).

\section{High-Performance Liquid Chromatography-Mass Spectrometry (HPLC-MS)}

Cerebrospinal fluid (CSF) samples were mixed with acetonitrile at room temperature, and then centrifuged at $10,000 \times \mathrm{g}$ for $5 \mathrm{~min}$. After that, the supernatant of each sample was collected.

Samples were analyzed by LC-MS using an LC-20AD HPLC (Shimadzu, Japan) coupled to an API 400 triple quadrupole MS (AB Sciex, Beijing, China). HPLC separation of physcion was carried out on a Venusil C18 Plus column $(50 \times$ 
$2.1 \mathrm{~mm}, 5 \mu \mathrm{m}$ particles diameter) manufactured by Agela (Torrance, CA, USA). Mobile phase A was ultrapure water, and mobile phase $\mathrm{B}$ was acetonitrile. The flow rate was $0.8 \mathrm{~mL} / \mathrm{min}$ and the elution gradient were programmed as following: initial, $0 \% \mathrm{~B}$; $0.01 \mathrm{~min}, 50 \% \mathrm{~B} ; 0.5 \mathrm{~min}, 95 \% \mathrm{~B}$; $1.3 \mathrm{~min}, 95 \% \mathrm{~B} ; 1.4 \mathrm{~min}, 50 \% \mathrm{~B} ; 2 \mathrm{~min}$, stop. The MS detector was equipped with the electrospray ionization and operated in the negative ion mode and MRM scan type. Operational electrospray ionization parameters were: spray voltage $-4200 \mathrm{~V}$, turbo ion spray temperature $500^{\circ} \mathrm{C}$.

\section{Statistical Analysis}

Each group was performed at least three independent experiments. Statistical analysis was analyzed by GraphPad Prism v7.0 (GraphPad Software, Inc., La Jolla, CA, USA) and all data were presented as the mean \pm standard deviation (SD). The statistical analysis of the results was analyzed with a one-way analysis of variance (ANOVA) followed by Tukey's test. $\mathrm{P}<0.05$ was considered significant.

\section{Results}

\section{Physcion Protects Cell Against OGD/ R-Induced Neuronal Injury}

CCK-8 assay was firstly used to assess the effect of physcion on the viability of SH-SY5Y cells. As shown in Figure 1A, $20 \mu \mathrm{M}$ physcion significantly increased the viability of $\mathrm{SH}$ SY5Y cells, while physcion $(10,40$ or $80 \mu \mathrm{M})$ had a limited effect on the viability of SH-SY5Y cells. In addition, OGD/ $\mathrm{R}$ remarkably reduced the viability, and increased the release of LDH in SH-SY5Y cells (Figure 1B and C). Conversely, physcion $(10,20$ or $40 \mu \mathrm{M})$ reversed OGD/R-induced SHSY5Y cell injury by increasing cell viability and reducing the release of LDH (Figure 1B and C). Moreover, OGD/R significantly induced the apoptosis of SH-SY5Y cells;
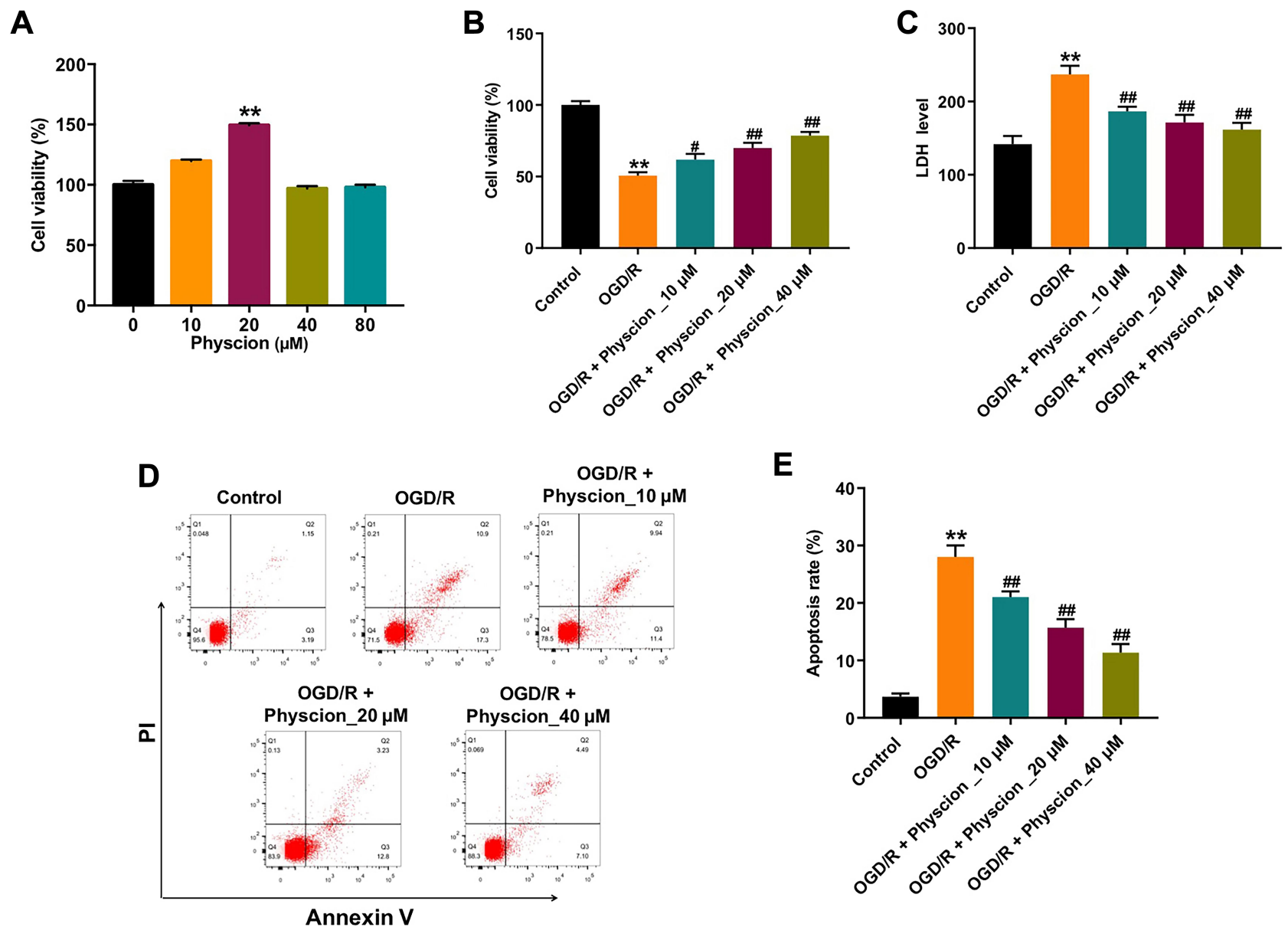

E

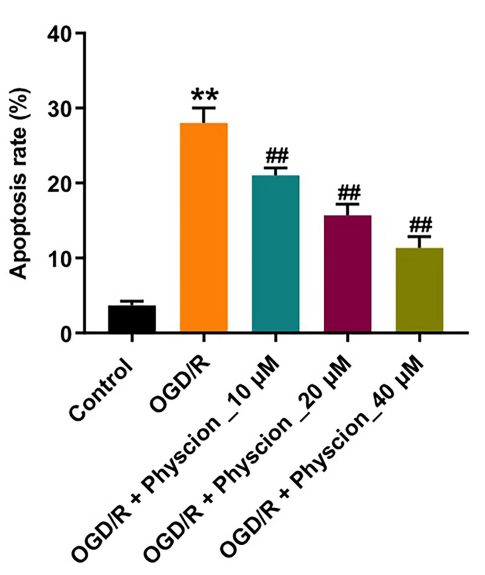

Figure I Physcion protects cell against OGD/R-induced neuronal injury. (A) SH-SY5Y cells were treated with $0,10,20,40$ or $80 \mu M$ physcion for 24 h. Cell viability was determined using CCK-8 assay. (B) SH-SY5Y cells were exposed to OGD for $4 \mathrm{~h}$, and then reoxygenated in the presence of I0, 20, or $40 \mu \mathrm{M}$ physcion for $24 \mathrm{~h}$. Cell viability was determined using CCK-8 assay. (C) Cell cytotoxicity was determined using LDH release assay. (D and E) Apoptotic cells were measured by flow cytometry. $* *$ P $<0.01$ compared with control group; ${ }^{\#} \mathrm{P}<0.05,{ }^{\#} \mathrm{P}<0.0 \mathrm{I}$ compared with $\mathrm{OGD} / \mathrm{R}$ group.

Abbreviations: OGD/R, oxygen-glucose deprivation/reperfusion; $\mathrm{PI}$, propidium iodide. 
however, OGD/R-induced apoptosis in SH-SY5Y cells was notably reversed by physcion $(10,20$ or $40 \mu \mathrm{M}$ ) (Figure $1 \mathrm{D}$ and E). All these data demonstrated that physcion could protect cell OGD/R-induced neuronal injury.

\section{Physcion Attenuates OGD/R-Induced Oxidative Stress in SH-SY5Y Cells}

Next, we investigated the effects of physcion on OGD/ R-induced oxidative stress in SH-SY5Y cells by detecting intracellular ROS generation. As shown in Figure 2A and $\mathrm{B}$, physcion $(40 \mu \mathrm{M})$ treatment significantly reduced OGD/R-induced ROS production in SH-SY5Y cells. In addition, OGD/R markedly increased MDA level, and reduced SOD and GSH levels in SH-SY5Y cells; however, these changes were markedly reversed by physcion treatment during the course of reperfusion (Figure 2C-E).
These results indicated that physcion could attenuate OGD/R-induced oxidative stress in SH-SY5Y cells.

\section{Physcion Suppresses OGD/R-Induced Inflammatory Response in SH-SY5Y Cells}

To investigate whether physcion could suppress the inflammatory response in SH-SY5Y cells in the condition of OGD/ $\mathrm{R}$ insult, the levels of the pro-inflammatory factors were detected. As shown in Figure 3A-E, OGD/R notably increased the levels of IL-1 $\beta$, TNF- $\alpha$, IL- 6 and MCP-1, and reduced IL-10 level in the supernatants of SH-SY5Y cells; however, these OGD/R-induced changes were significantly reversed by physcion treatment during the course of reperfusion. These data suggested that physcion could suppress OGD/R-induced inflammatory response in SH-SY5Y cells.
A

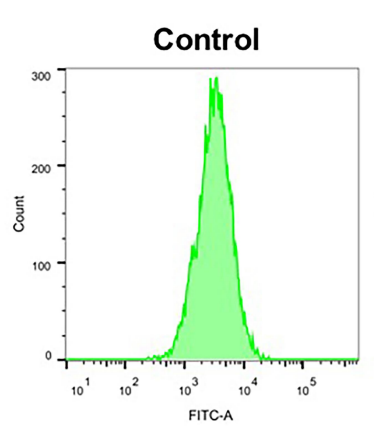

OGD/R

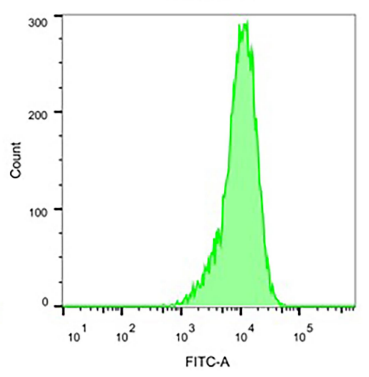

OGD/R + Physcion_40 $\mu \mathrm{M}$

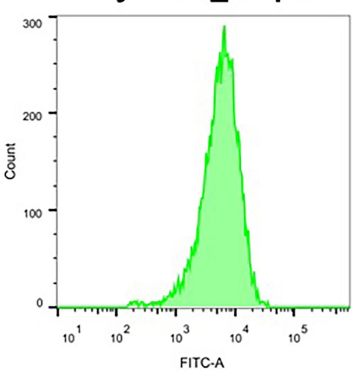

FITC.A

B

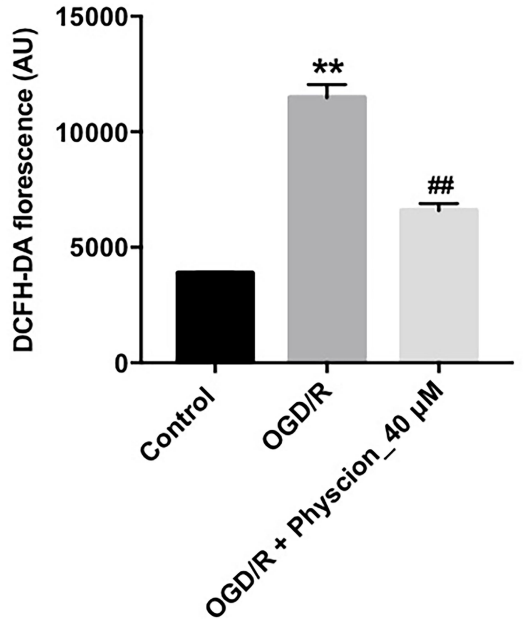

C

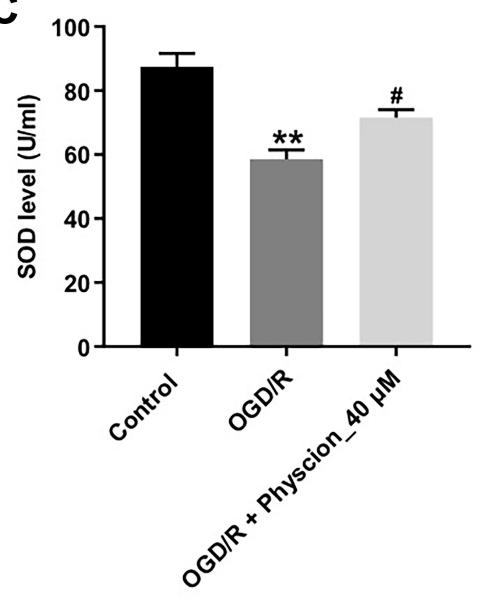

D

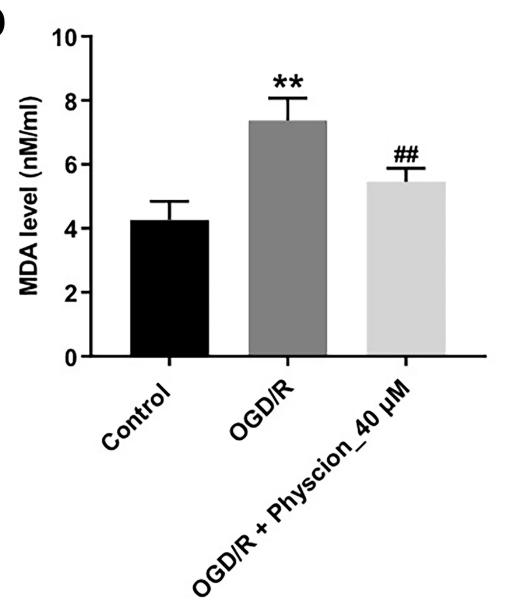

E

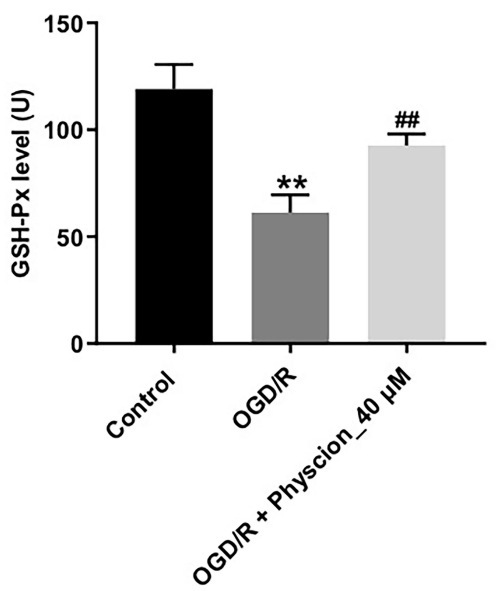

Figure 2 Physcion attenuates OGD/R-induced oxidative stress in SH-SY5Y cells. SH-SY5Y cells were exposed to OGD for $4 \mathrm{~h}$, and then reoxygenated in the presence of 40 $\mu \mathrm{M}$ physcion for $24 \mathrm{~h}$. (A and B) Intracellular ROS generation was assessed by flow cytometry. (C-E) Levels of SOD, MDA and GSH-Px in cells were detected with ELISA. *** $\mathrm{P}<0.01$ compared with control group; ${ }^{\#} \mathrm{P}<0.05,{ }^{\# \#} \mathrm{P}<0.01$ compared with $\mathrm{OGD} / \mathrm{R}$ group.

Abbreviations: DCFH-DA, 2,7-dichlorofluorescein diacetate; GSH-px, glutathione peroxidase; MDA, malondialdehyde; OGD/R, oxygen-glucose deprivation/reperfusion; SOD, superoxide dismutase. 

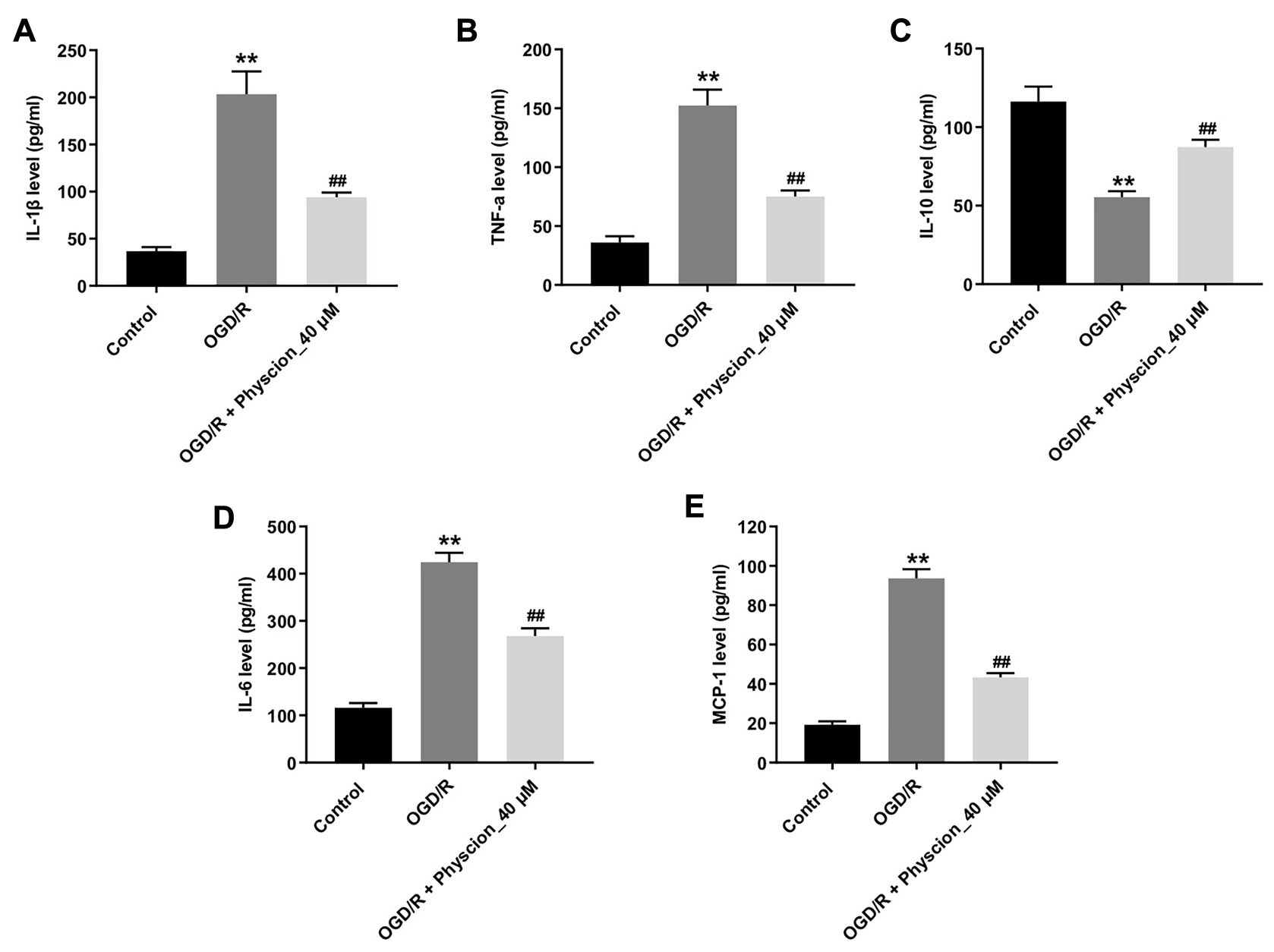

Figure 3 Physcion suppresses OGD/R-induced inflammatory response in SH-SY5Y cells. SH-SY5Y cells were exposed to OGD for $4 \mathrm{~h}$, and then reoxygenated in the presence of $40 \mu \mathrm{M}$ physcion for $24 \mathrm{~h}$. (A-E) Levels of IL-I $\beta$, TNF- $\alpha$, IL-IO, IL-6 and MCP-I in cells were detected with ELISA. **P<0.0I compared with control group; ${ }^{\prime \prime} \mathrm{P}<0.01$ compared with $\mathrm{OGD} / \mathrm{R}$ group.

Abbreviations: IL-10, interleukin-10; IL-I $\beta$, interleukin-I $\beta$; IL-6, interleukin-6; MCP-I, macrophage chemoattractant protein I; OGD/R, oxygen-glucose deprivation/ reperfusion; TNF- $\alpha$, tumor necrosis factor- $\alpha$.

\section{Physcion Attenuates OGD/R-Induced Injury in SH-SY5Y Cells via Inhibiting the TLR4/NF-кB Pathway}

Evidence has shown that suppression of TLR4/NF- $\mathrm{BB}$ signaling could improve cerebral $\mathrm{I} / \mathrm{R}$ injury. ${ }^{18}$ Thus, to explore whether physcion protected OGD/R-induced neuronal cell injury via mediating the TLR4/NF- $\kappa \mathrm{B}$ pathway, Western blot assay was performed. As indicated in Figure 4A-D, the expressions of TLR4, p-p65 and $\mathrm{p}$-IкB were notably upregulated in OGD/R-treated SH-SY5Y cells; however, these OGD/R-induced changes were significantly reversed by physcion treatment in the course of reperfusion. These data indicated that physcion could attenuate OGD/R-induced injury in SH-SY5Y cells via inhibiting the TLR4/NF- $\mathrm{KB}$ pathway.

\section{Physcion Protects Rats Against Brain Damage After I/R via Inhibiting the TLR4/ NF- $\kappa$ B Pathway}

A rat model of focal cerebral ischemia was established to explore the role of physcion in IS in vivo. To explore whether physcion is capable of crossing the blood-brain barrier (BBB) in rat brain, HPLC-MS/MS was performed to measure the level of physcion in CSF. The results indicated that the concentration of physcion in rat CSF was $196 \mathrm{ng} / \mathrm{mL}$ in $\mathrm{I} / \mathrm{R}+$ physcion_40 mg/kg group, confirming that physcion was able to penetrate the BBB (Supplementary Figure 1A and $\underline{B}$ ). In addition, TTC staining showed that the rats in $\mathrm{I} / \mathrm{R}$ group exhibited marked areas of infarction (Figure 5A and B, Supplementary Table 1). However, physcion treatment obviously decreased areas of infarction compared to $I / R$ group (Figure 5A and B, Supplementary Table 1). 

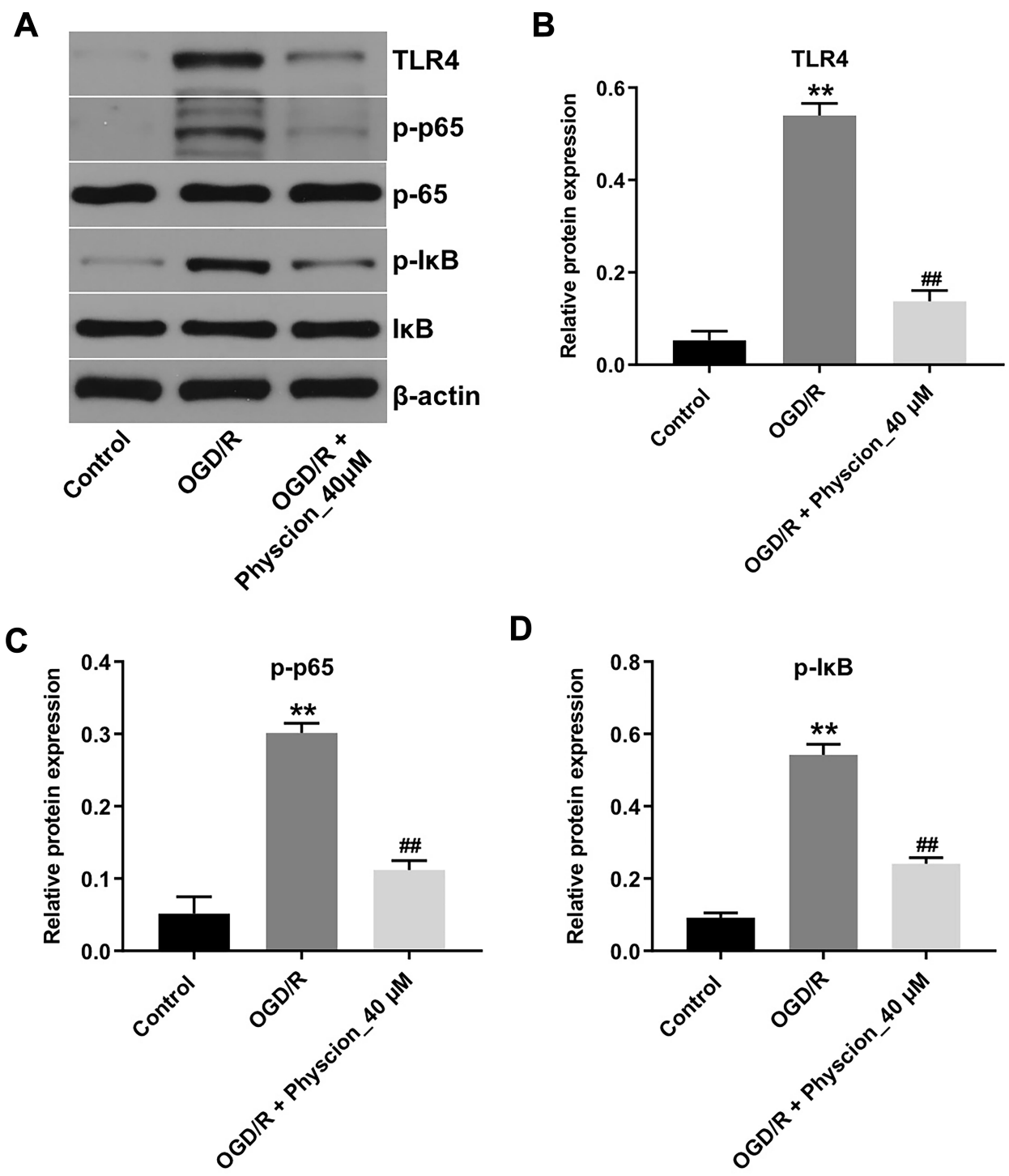

Figure 4 Physcion attenuates OGD/R-induced injury in SH-SY5Y cells via inhibiting the TLR4/p65 pathway. SH-SY5Y cells were exposed to OGD for 4 h, and then reoxygenated in the presence of $40 \mu \mathrm{M}$ Oroxylin A for $24 \mathrm{~h}$. (A) Expression levels of TLR4, p-p65, p-lкB in SH-SY5Y cells were detected with Western blotting. (B-D) The relative expressions of TLR4, $\mathrm{p}-\mathrm{p} 65, \mathrm{p}-\mathrm{I} / \mathrm{B}$ in cells were quantified via normalization to $\beta$-actin, $\mathrm{p} 65$ and $\mathrm{I} \mathrm{kB}$. ${ }^{* * P} \mathrm{P}<0.01$ compared with control group; ${ }^{\#} \mathrm{P}<0.01$ compared with OGD/R group.

Abbreviations: OGD/R, oxygen-glucose deprivation/reperfusion; p-IKB, phospho-IKB; p65, NF-kB p65; p-p65, phospho-NF-kB p65; TLR4, toll-like receptor 4.

Meanwhile, physcion significantly reduced the brain water content and the NSS in $\mathrm{I} / \mathrm{R}$ rats, compared to $\mathrm{I} / \mathrm{R}$ group (Figure 5C and D, Supplementary Tables 2 and $\underline{3}$ ). Moreover, HE and TUNEL assays showed that physcion treatment had protective effects against tissue injury in the brain slice of rats in the condition of cerebral $I / R$ injury (Figure 5E and F, Supplementary Table 4).

Furthermore, the expressions of TLR4, p-p65 and p-IкB were markedly upregulated in whole brain tissue of I/R rats; however, these changes were reversed by physcion treatment in the course of reperfusion (Figure 6A-D). All these data illustrated that physcion could protect rats against brain injury after cerebral $\mathrm{I} / \mathrm{R}$ via inhibiting the TLR4/NF- $\mathrm{KB}$ pathway.

\section{Discussion}

It has been shown that physcion plays a therapeutic role in central nervous system (CNS) diseases, including cerebral IS. ${ }^{19}$ In this study, we performed a study to investigate the regulatory effect of physcion in cerebral $\mathrm{I} / \mathrm{R}$ injury. Our data indicated that $\mathrm{OGD} / \mathrm{R}$ significantly reduced the viability, increased the release of LDH and cell apoptosis in SH-SY5Y cells; however, these effects were reversed by the treatment with physcion $(10,20$ or $40 \mu \mathrm{M})$. 

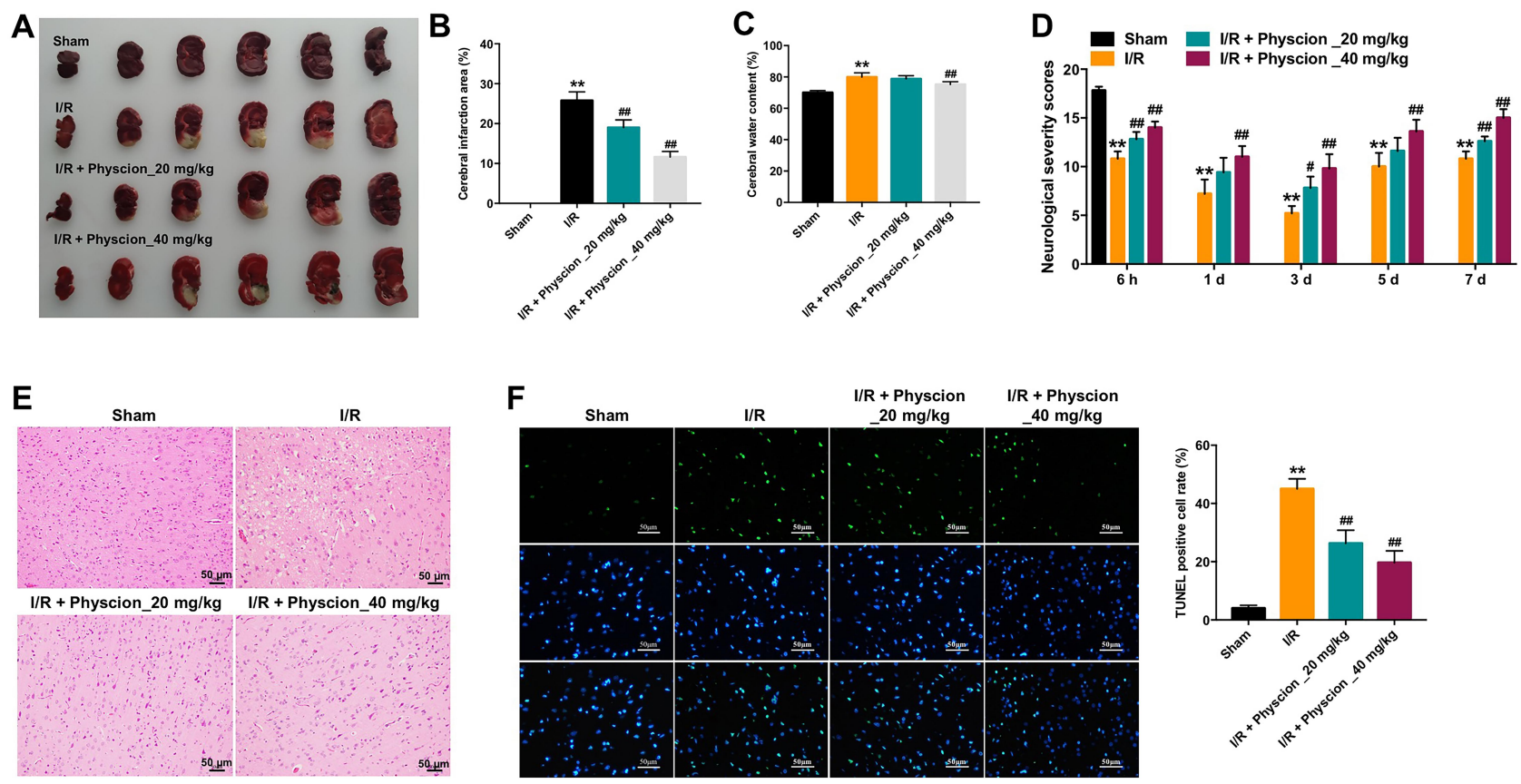

Figure 5 Physcion protects rats against brain damage after I/R. (A and B) TTC staining was applied to assess infarct area (magenta: healthy tissue; white: damaged tissue). (C) Cerebral water content was calculated. (D) Neurobehavioral tests were performed at 6 h, I, 3, 5 or 7 days after MCAO. Neurological severity scores (NSS). (E) HE staining assay was performed to detect brain tissue injury in I/R rats (magnification $\times 200$ ). (F) TUNEL assay was performed to detect cell apoptosis in I/R rats challenged with MCAO (magnification $\times 400$ ). ${ }^{* * P}<0.01$ compared with sham group; ${ }^{\#} \mathrm{P}<0.05,{ }^{\# \#} \mathrm{P}<0.01$ compared with $\mathrm{I} / \mathrm{R}$ group.

Abbreviations: I/R, ischemia-reperfusion; TUNEL, terminal deoxynucleotidyl transferase dUTP nick end labeling.

Importantly, $40 \mu \mathrm{M}$ physcion exhibited the most pronounced effect in preventing OGD/R-induced SH-SY5Y cell injury. In addition, our results indicated that physcion could attenuate $\mathrm{I} / \mathrm{R}$-mediated neuron injury via attenuating oxidative stress, inflammatory response, and inactivating the TLR4/NF-кB pathway. Consistently, Li et al found that $26.4 \mathrm{mg} / \mathrm{kg}$ physcion exhibited anti-ischemic effect in rats with cerebral ischemia. ${ }^{13}$ In this study, we found that physcion $(20$ or $40 \mathrm{mg} / \mathrm{kg}$ ) protected against cerebral $\mathrm{I} / \mathrm{R}$ injury in rats via inactivating the TLR4/NF- $\mathrm{kB}$ pathway. These data indicated that physcion could protect against cerebral $\mathrm{I} / \mathrm{R}$ injury in vitro and in vivo via inhibition of TLR4/NF-kB signaling.

ROS generation is implicated in the $I / R$ injury, increased ROS could lead to neuronal cell death. ${ }^{16,20}$ In addition, oxidative damage can consume intracellular
A

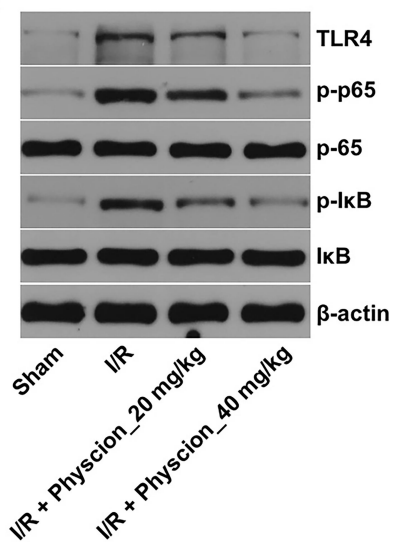

B

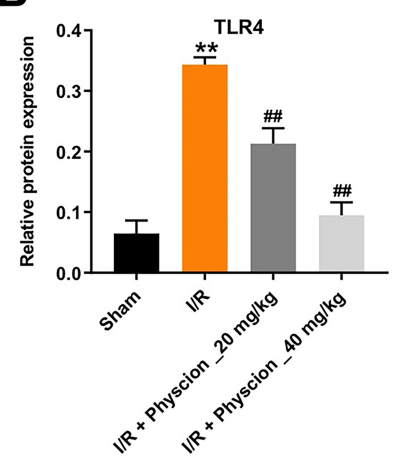

C

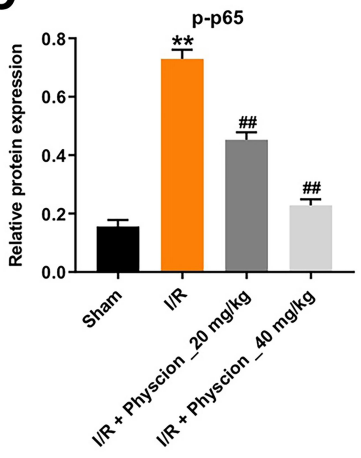

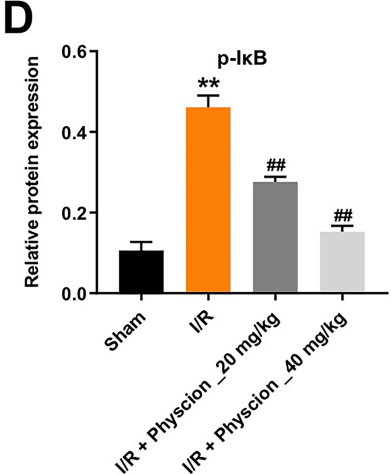

Figure 6 Physcion protects rats against brain damage after I/R via inhibiting the TLR4/p65 pathway. (A) Expression levels of TLR4, P-p65, p-IkB in the whole brain tissues of I/R rats were detected with Western blotting. (B-D) The relative expressions of TLR4, $p-p 65, p-I \kappa B$ in the whole brain tissues of $I / R$ rats were quantified via normalization to $\beta$-actin, $\mathrm{p} 65$ and IKB. ${ }^{* * P} \mathrm{P}<0.01$ compared with sham group; ${ }^{\# \#} \mathrm{P}<0.01$ compared with $\mathrm{I} / \mathrm{R}$ group.

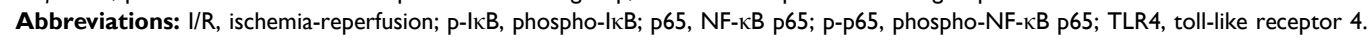


antioxidants (GSH and SOD), and lead to the production of MDA. ${ }^{21,22}$ Evidences have shown that cerebral I/R injury could increase ROS generation and induce oxidative stress by suppressing the activities of antioxidant biomarkers, ${ }^{23,24}$ which were again confirmed in the current study. Sun et al found that cannabidiol can protect hippocampal neurons against OGD/R-induced injury via attenuating oxidative stress. ${ }^{25} \mathrm{Wu}$ et al indicated that curcumin could attenuate OGD/R-induced injury in SH-SY5Y cells via increasing the levels of SOD and GSH. ${ }^{26}$ In this study, we found that physcion could reduce OGD/R-induced oxidative stress in SH-SY5Y cells, as shown by the decreased generations of ROS and MDA, and the increased activities of GSH and SOD. These data suggested that physcion could protect SH-SY5Y cells against OGD/R-induced oxidative stress. In addition, consistent with previous studies, ${ }^{27,28}$ we found that OGD/R insult significantly reduced cell viability via inducing apoptosis, which was reversed by the administration of physcion.

Inflammatory mediators are key contributing factors in cerebral ischemic injury. ${ }^{29,30}$ It has been shown that TLR4 contributes to I/R-induced inflammatory response. ${ }^{31}$ Our data indicated that the expression of TLR4 was increased in SH-SY5Y cells exposed to OGD/R and in brain tissues of rats subjected to I/R injury. In addition, NF- $\mathrm{kB}$ is an essential transcription factor that plays an important role in cell growth and inflammation. ${ }^{9,32} \mathrm{NF}-\mathrm{kB}$ exists in cytoplasm as an inactive dimeric form binding to IKB. ${ }^{31}$ TLR4/MyD88 signaling can phosphorylate $\mathrm{I} \kappa \mathrm{B}$, and then release the active form of NF- $\kappa B$ (NF- $\kappa B$ translocation from the cytoplasm to the nucleus). ${ }^{33} \mathrm{NF}-\mathrm{kB}$ participates in the pathological process of IS, which can be triggered by ROS, and several inflammatory cytokines. ${ }^{32}$ Moreover, it also has the ability to regulate the production and release of pro-inflammatory factors, including IL-1 $\beta$, IL- 6 , and TNF- $\alpha$ in neurons. ${ }^{34} \mathrm{Ye}$ et al found that meisoindigo protected against cerebral $\mathrm{I} / \mathrm{R}$ injury by inhibiting TLR4/NF- $\mathrm{KB}$ signaling pathway. ${ }^{18} \mathrm{Zhu}$ et al indicated that berberine attenuated $\mathrm{I} / \mathrm{R}$ injury via inhibiting NF- $\mathrm{KB}$ nuclear translocation. ${ }^{7}$ Meanwhile, physcion was reported to possess an anti-inflammatory effect in LPSstimulated HepG2 cells. ${ }^{35}$ In this study, we found that TLR4, p-p65, and p-IкB proteins, and inflammatory factors, including IL- $1 \beta$, TNF- $\alpha$, IL-6 and MCP-1, were all upregulated in SH-SY5Y cells during OGD/R injury. However, these OGD/R-induced changes were significantly reversed by physcion treatment in the course of reperfusion, indicating that physcion-inhibiting inflammatory responses were likely to contribute to attenuating cerebral $\mathrm{I} / \mathrm{R}$ injury.
Collectively, physcion attenuated OGD/R-induced inflammatory response in SH-SY5Y cells via inhibition of TLR4/ NF- $\kappa B$ pathway. Furthermore, wang et al found that curcumin could reduce the apoptosis and inflammation of $\mathrm{PC} 12$ cells after $\mathrm{OGD} / \mathrm{R}$ insult via inactivating the TLR4/NF- $\mathrm{KB}$ pathway. ${ }^{36}$ In addition, Gao et al indicated that pterostilbene could inhibit oxidative stress in a mouse model of acute renal I/R injury via inhibition of TLR4/NF- $\mathrm{KB}$ pathway. ${ }^{37}$ These data indicated that physcion could attenuate the neuronal apoptosis, oxidative stress and inflammatory response induced by $\mathrm{I} / \mathrm{R}$ injury via inactivating the TLR4/NF- $\mathrm{KB}$ signaling.

\section{Conclusion}

In summary, the present study demonstrated that physcion could protect against rats' cerebral $\mathrm{I} / \mathrm{R}$ injury via inhibition of the TLR4/NF- $\kappa B$ signaling. Our findings might provide a potential therapeutic strategy for IS.

\section{Acknowledgment}

Xiaobo Dong and Lei Wang are co-first authors for this study.

\section{Funding}

This study received funding from the State Administration of Traditional Chinese Medicine of the Special Scientific Research on the Business Construction of National TCM Clinical Base (No. JDZX2015043) and Key Research Projects of Beijing University of Chinese Medicine in 2020 (No. 2020-JYB-ZDGG-129).

\section{Disclosure}

The authors declare no competing financial interests.

\section{References}

1. Wicha P, Tocharus J, Janyou A, et al. Hexahydrocurcumin protects against cerebral ischemia/reperfusion injury, attenuates inflammation, and improves antioxidant defenses in a rat stroke model. PLoS One. 2017;12(12):e0189211. doi:10.1371/journal.pone.0189211

2. Wu R, Li X, Xu P, et al. TREM2 protects against cerebral ischemia/ reperfusion injury. Mol Brain. 2017;10(1):20. doi:10.1186/s13041-0170296-9

3. Gong L, Tang Y, An R, et al. RTN1-C mediates cerebral ischemia/reperfusion injury via ER stress and mitochondria-associated apoptosis pathways. Cell Death Dis. 2017;8(10):e3080. doi:10.1038/cddis.2017.465

4. Chen Z, Yang J, Zhong J, et al. MicroRNA-193b-3p alleviates focal cerebral ischemia and reperfusion-induced injury in rats by inhibiting 5-lipoxygenase expression. Exp Neurol. 2020;327:113223. doi:10.1016/j.expneurol.2020.113223

5. Jung JE, Kim GS, Chen H, et al. Reperfusion and neurovascular dysfunction in stroke: from basic mechanisms to potential strategies for neuroprotection. Mol Neurobiol. 2010;41(2-3):172-179. doi:10.1007/s12035-010-8102-z 
6. Harston GW, Rane N, Shaya G, et al. Imaging biomarkers in acute ischemic stroke trials: a systematic review. AJNR Am J Neuroradiol. 2015;36(5):839-843. doi:10.3174/ajnr.A4208

7. Zhu JR, Lu HD, Guo C, et al. Berberine attenuates ischemiareperfusion injury through inhibiting HMGB1 release and NF- $\mathrm{NB}$ nuclear translocation. Acta Pharmacol Sin. 2018;39(11):1706-1715. doi:10.1038/s41401-018-0160-1

8. Hwang CJ, Yun HM, Jung YY, et al. Reducing effect of IL-32 $\alpha$ in the development of stroke through blocking of NF- $\mathrm{B}$, but enhancement of STAT3 pathways. Mol Neurobiol. 2015;51(2):648-660. doi: $10.1007 /$ s 12035-014-8739-0

9. Liu H, Wu X, Luo J, et al. Pterostilbene attenuates astrocytic inflammation and neuronal oxidative injury after ischemia-reperfusion by inhibiting NF-кB phosphorylation. Front Immunol. 2019;10:2408. doi:10.3389/fimmu.2019.02408

10. Pang MJ, Yang Z, Zhang XL, et al. Physcion, a naturally occurring anthraquinone derivative, induces apoptosis and autophagy in human nasopharyngeal carcinoma. Acta Pharmacol Sin. 2016;37 (12):1623-1640. doi:10.1038/aps.2016.98

11. Guo S, Feng B, Zhu R, Ma J, Wang W. Preparative isolation of three anthraquinones from rumex japonicus by high-speed counter-current chromatography. Molecules. 2011;16(2):1201-1210. doi:10.3390/ molecules16021201

12. Han YT, Chen XH, Gao H, Ye JL, Wang CB. Physcion inhibits the metastatic potential of human colorectal cancer SW620 cells in vitro by suppressing the transcription factor SOX2. Acta Pharmacol Sin. 2016;37(2):264-275. doi:10.1038/aps.2015.115

13. Li RR, Liu XF, Feng SX, et al. Pharmacodynamics of five anthraquinones (aloe-emodin, emodin, rhein, chrysophanol, and physcion) and reciprocal pharmacokinetic interaction in rats with cerebral ischemia. Molecules. 2019;24(10). doi:10.3390/molecules24101898.

14. Feng SX, Li JS, Qu LB, Shi YM, Zhao D. Comparative pharmacokinetics of five rhubarb anthraquinones in normal and thrombotic focal cerebral ischemia-induced rats. Phytother Res. 2013;27 (10):1489-1494. doi:10.1002/ptr.4890

15. Yan H, Rao J, Yuan J, et al. Long non-coding RNA MEG3 functions as a competing endogenous RNA to regulate ischemic neuronal death by targeting miR-21/PDCD4 signaling pathway. Cell Death Dis. 2017;8(12):3211. doi:10.1038/s41419-017-0047-y

16. Lee JH, Jeong JH, Jeong YG, et al. Platycarya strobilacea leaf extract protects mice brain with focal cerebral ischemia by antioxidative property. Anat Cell Biol. 2019;52(4):486-497. doi:10.5115/ acb.19.141

17. Chen J, Li Y, Wang L, et al. Therapeutic benefit of intravenous administration of bone marrow stromal cells after cerebral ischemia in rats. Stroke. 2001;32(4):1005-1011. doi:10.1161/01. STR.32.4.1005

18. Ye Y, Jin $\mathrm{T}$, Zhang $\mathrm{X}$, et al. Meisoindigo protects against focal cerebral ischemia-reperfusion injury by inhibiting NLRP3 inflammasome activation and regulating microglia/macrophage polarization via TLR4/NF-кB signaling pathway. Front Cell Neurosci. 2019;13:553. doi:10.3389/fncel.2019.00553

19. Li X, Chu S, Liu Y, Chen N. Neuroprotective effects of anthraquinones from rhubarb in central nervous system diseases. Evid Based Complement Alternat Med. 2019;2019:3790728.

20. Finkel T, Holbrook NJ. Oxidants, oxidative stress and the biology of ageing. Nature. 2000;408(6809):239-247. doi:10.1038/35041687

21. Zhang Y, Cheng Z, Wang C, et al. Neuroprotective effects of kukoamine a against radiation-induced rat brain injury through inhibition of oxidative stress and neuronal apoptosis. Neurochem Res. 2016;41 (10):2549-2558. doi:10.1007/s11064-016-1967-0

22. Yang CY, Lin MT. Oxidative stress in rats with heatstroke-induced cerebral ischemia. Stroke. 2002;33(3):790-794. doi:10.1161/ hs0102.100208
23. Sun MS, Jin $H$, Sun $X$, et al. Free radical damage in ischemia-reperfusion injury: an obstacle in acute ischemic stroke after revascularization therapy. Oxid Med Cell Longev. 2018;2018:3804979. doi:10.1155/2018/3804979

24. Kalogeris T, Bao Y, Korthuis RJ. Mitochondrial reactive oxygen species: a double edged sword in ischemia/reperfusion vs preconditioning. Redox Biol. 2014;2:702-714. doi:10.1016/j. redox.2014.05.006

25. Sun S, Hu F, Wu J, Zhang S. Cannabidiol attenuates OGD/R-induced damage by enhancing mitochondrial bioenergetics and modulating glucose metabolism via pentose-phosphate pathway in hippocampal neurons. Redox Biol. 2017;11:577-585. doi:10.1016/j. redox.2016.12.029

26. Wu L, Jiang C, Kang Y, et al. Curcumin exerts protective effects against hypoxia-reoxygenation injury via the enhancement of apurinic/apyrimidinic endonuclease 1 in SH-SY5Y cells: involvement of the PI3K/AKT pathway. Int J Mol Med. 2020;45(4):993-1004. doi:10.3892/ijmm.2020.4483

27. Zhang Y, Zhang Y, Jin XF, et al. The role of astragaloside IV against cerebral ischemia/reperfusion injury: suppression of apoptosis via promotion of P62-LC3-autophagy. Molecules. 2019;24(9).

28. Zhao L, Zhuang J, Wang Y, et al. Propofol ameliorates H9c2 cells apoptosis induced by oxygen glucose deprivation and reperfusion injury via inhibiting high levels of mitochondrial fusion and fission. Front Pharmacol. 2019;10:61. doi:10.3389/fphar.2019.00061

29. Del Zoppo G, Ginis I, Hallenbeck JM, et al. Inflammation and stroke: putative role for cytokines, adhesion molecules and iNOS in brain response to ischemia. Brain Pathol. 2000;10(1):95-112. doi:10.1111/ j.1750-3639.2000.tb00247.x

30. Liu H, Wei X, Kong L, et al. NOD2 is involved in the inflammatory response after cerebral ischemia-reperfusion injury and triggers NADPH oxidase 2-derived reactive oxygen species. Int J Biol Sci. 2015;11(5):525-535. doi:10.7150/ijbs.10927

31. Zhang W, Song J, Li W, et al. Salvianolic acid D alleviates cerebral ischemia-reperfusion injury by suppressing the cytoplasmic translocation and release of HMGB1-triggered NF- $\kappa \mathrm{B}$ activation to inhibit inflammatory response. Mediators Inflamm. 2020;2020:9049614. doi:10.1155/2020/9049614

32. Ridder DA, Schwaninger M. NF-kappaB signaling in cerebral ischemia. Neuroscience. 2009;158(3):995-1006. doi:10.1016/j. neuroscience.2008.07.007

33. Su X, Wang H, Zhao J, Pan H, Mao L. Beneficial effects of ethyl pyruvate through inhibiting high-mobility group box 1 expression and TLR4/NF- $\kappa \mathrm{B}$ pathway after traumatic brain injury in the rat. Mediators Inflamm. 2011;2011:807142. doi:10.1155/2011/807142

34. Jung HS, Joo JD, Kim DW, et al. Effect of milrinone on the inflammatory response and NF-kB activation in renal ischemia-reperfusion injury in mice. Korean $J$ Anesthesiol. 2014;66(2):136-142. doi:10.4097/kjae.2014.66.2.136

35. Selim NM, Elgazar AA, Abdel-Hamid NM, et al. Chrysophanol, physcion, hesperidin and curcumin modulate the gene expression of pro-inflammatory mediators induced by LPS in HepG2: in silico and molecular studies. Antioxidants (Basel). 2019;8(9).

36. Wang C, Yang YH, Zhou L, et al. Curcumin alleviates OGD/ $\mathrm{R}$-induced PC12 cell damage via repressing CCL3 and inactivating TLR4/MyD88/MAPK/NF- $\mathrm{KB}$ to suppress inflammation and apoptosis. J Pharm Pharmacol. 2020;72(9):1176-1185. doi:10.1111/ jphp. 13293

37. Gao D, Jing S, Zhang Q, Wu G. Pterostilbene protects against acute renal ischemia reperfusion injury and inhibits oxidative stress, inducible nitric oxide synthase expression and inflammation in rats via the Toll-like receptor 4/nuclear factor- $\kappa \mathrm{B}$ signaling pathway. Exp Ther Med. 2018;15(1):1029-1035. doi:10.3892/etm.2017.5479 


\section{Publish your work in this journal}

Drug Design, Development and Therapy is an international, peerreviewed open-access journal that spans the spectrum of drug design and development through to clinical applications. Clinical outcomes, patient safety, and programs for the development and effective, safe, and sustained use of medicines are a feature of the journal, which has also been accepted for indexing on PubMed Central. The manuscript management system is completely online and includes a very quick and fair peer-review system, which is all easy to use. Visit http://www. dovepress.com/testimonials.php to read real quotes from published authors.

Submit your manuscript here: https://www.dovepress.com/drug-design-development-and-therapy-journal 